\title{
Adult Olfactory Bulb Neurogenesis
}

\author{
Pierre-Marie Lledo and Matt Valley \\ Pasteur Institute, the Laboratory for Perception and Memory, CNRS Unit Genes, \\ Synapses \& Cognition, UMR 3571, 75724 Paris Cedex 15, France \\ Correspondence: pierre-marie.Iledo@pasteur.fr
}

Most organisms use their olfactory system to detect and analyze chemical cues from the external world to guide essential behaviors. From worms to vertebrates, chemicals are detected by odorant receptors expressed by olfactory sensory neurons, which in vertebrates send an axon to the primary processing center called the olfactory bulb (OB). Within the $\mathrm{OB}$, sensory neurons form excitatory synapses with projection neurons and with inhibitory interneurons. Thus, because of complex synaptic interactions, the output of a given projection neuron is determined not only by the sensory input, but also by the activity of local inhibitory interneurons that are regenerated throughout life in the process of adult neurogenesis. Herein, we discuss how it is optimized and why.

$\mathrm{R}^{\mathrm{e}}$ ecent studies have provided clues about how new neurons incorporate into preexisting networks, the factors that govern their survival or death, and how the unique physiology of these new neurons influences olfactory circuit function. We know that external influences modulate the process of adult neurogenesis at various stages. More recently, it has become clear that higher brain states also shape the degree of adult neurogenesis. Thus, this process is probably flexible, allowing brain performance to be optimized for both environmental and internal challenges. But optimized how? And why?

Here, we will detail how the new interneurons provide experience-induced plasticity within the olfactory bulb (OB). In particular, we will discuss how the survival of newly generated neurons is not only highly sensitive to sensory inputs, but also to behavioral context. We also discuss how neurogenesis may finely tune the function of the OB's neural network, opti- mizing the processing of sensory information. Adult neurogenesis maintains continual turnover of bulbar interneurons that offer a large spectrum of various computational functions. In this way, adult neurogenesis may be one mechanism that can improve olfactory performance in specific contexts. This review brings together recent descriptions of newly generated neurons in the $\mathrm{OB}$ and emerging principles concerning their function. This review, concentrating on the mammalian olfactory system, highlights adult neurogenesis as part of the brain's arsenal for dealing with an environment and internal brain states that are constantly changing.

\section{STABILITY VERSUS FLEXIBILITY: A QUANDARY FOR ADULT NEURONAL CIRCUITS}

It has become clear over the past decades that new neurons are continually generated in the

Editors: Fred H. Gage, Gerd Kempermann, and Hongjun Song

Additional Perspectives on Neurogenesis available at www.cshperspectives.org

Copyright (C) 2016 Cold Spring Harbor Laboratory Press; all rights reserved

Advanced Online Article. Cite this article as Cold Spring Harb Perspect Biol doi: 10.1101/cshperspect.a018945 
adult brain. Two regions- the $\mathrm{OB}$ and the dentate gyrus of the hippocampus-receive and continually integrate newly generated neurons throughout life (Ming and Song 2005). The molecular and cellular mechanisms by which these cells are generated, and by which they migrate to their target structures, are areas of intense research. A major hope is that this research will generate insights that will generalize to clinical approaches attempting to regenerate damaged or degraded portions of neural tissue. In addition, adult neurogenesis produces a unique circuit architecture in which cellular composition is in constant flux. This raises fundamental questions about how information processing can be maintained in the face of plasticity, and, thus, it is critical to know how neurogenesis influences the normal function of neuronal circuits (Aimone et al. 2011; Ming and Song 2011).

It is not understood why neurogenesis persists in some parts of the adult brain but not in others. Adult neurogenesis may simply be a recapitulation of embryogenesis, or it may be a unique feature of certain regions of the adult forebrain. Adult neurogenesis is restricted to two specific regions under normal conditions, but we do not know why or how these regions balance the need for plasticity with their need to maintain stable information processing. An explanation for the marked spatial restriction of adult neurogenesis may only emerge after comprehensive study of how aging alters embryonic neurogenesis throughout the central nervous system.

Is neurogenesis in the adult brain a constant, restorative process, or is it flexible, producing different numbers of neurons in different regions in response to environmental stimuli or internal needs? We also ask whether new neurons are generated in the adult brain to perform particular tasks, or whether they are generated as flexible units that can undertake whatever role their target structure most requires. Likewise, one might wonder whether neurogenesis acts directly, in a specific manner, on neuronal information processing or whether it acts indirectly by preparing host circuits for general experiences and increased general challenges. It is noteworthy that common concepts about the functional consequences of neurogenesis may emerge from exploration of functional differences between the $\mathrm{OB}$ and the hippocampus as well as between neurogenesis in the developing and mature brain.

A key conclusion drawn from the discovery of neuronal production in the mature brain is that adult neurogenesis is a mechanism by which the brain alters its structural organization and the functions of its circuitry. Adult neurogenesis may thus be considered among other forms of plasticity, including the addition and removal of synaptic contacts. What more does neuronal turnover brings to the brain's ability to adapt than synaptic plasticity alone? A host of recent evidence shows that neuronal replacement is not merely restorative; instead, adult neurogenesis is a dynamic form of neuronal plasticity that enables the brain to adapt to the constantly evolving interaction between environmental signals and the brain's internal reaction to these stimuli.

From a different perspective, the addition of newly generated neurons to adult circuitry raises questions about stability. In particular, how do new cells integrate into an already functioning system without disturbing its existing properties? The brain must preserve its circuitry and synaptic organization so that important information is not lost. At the same time, it must adapt its circuitry to learn, repair injuries, and deal with environmental changes. This dilemma between stability and plasticity (Abrous et al. 2005) is a matter of passionate debate that is far from being resolved. Central to these debates is the question of whether new neurons provide new capabilities to the circuit. There are studies suggesting that newly generated neurons in the adult $\mathrm{OB}$ and hippocampus are critical for various aspects of learning, memory, and sensory performance. This review will present more general issues concerning the plasticity conferred to adult circuits by newly generated cells and by neuronal replacement.

\section{THE FUNCTION OF INTERNEURONS IN THE OB}

It has become clear that newly generated interneurons integrate into the local inhibitory net- 
work of the OB throughout life. Most newly generated cells that reach the OB become granule cells $(\sim 94 \%)$, and the remainder become periglomerular cells $(\sim 4 \%)$ or astrocytes $(<2 \%)$. Granule cells make up the largest percentage of neurons in the $\mathrm{OB}$, and each granule cell contacts several hundred relay neurons (mitral and tufted cells; Fig. 1), which, in turn, contact many pyramidal cells in the piriform cortex (i.e., a part of the olfactory cortex). Thus, because bulbar neurogenesis occurs early in the olfactory pathway, its effect may also be strongly felt by downstream structures. To understand how the birth of interneurons may alter the function of the bulb and, consequently, information processing by downstream brain areas, it is necessary to consider first the general function of interneurons within the OB.

Olfactory information is genetically organized into a simple spatial map as it enters the $\mathrm{OB}$ and activates functionally segregated synaptic structures called glomeruli. However, this simple map is radically transformed by the interactions between interneurons and mitral and tufted output neurons. Computationally, this transformation includes several operations in-

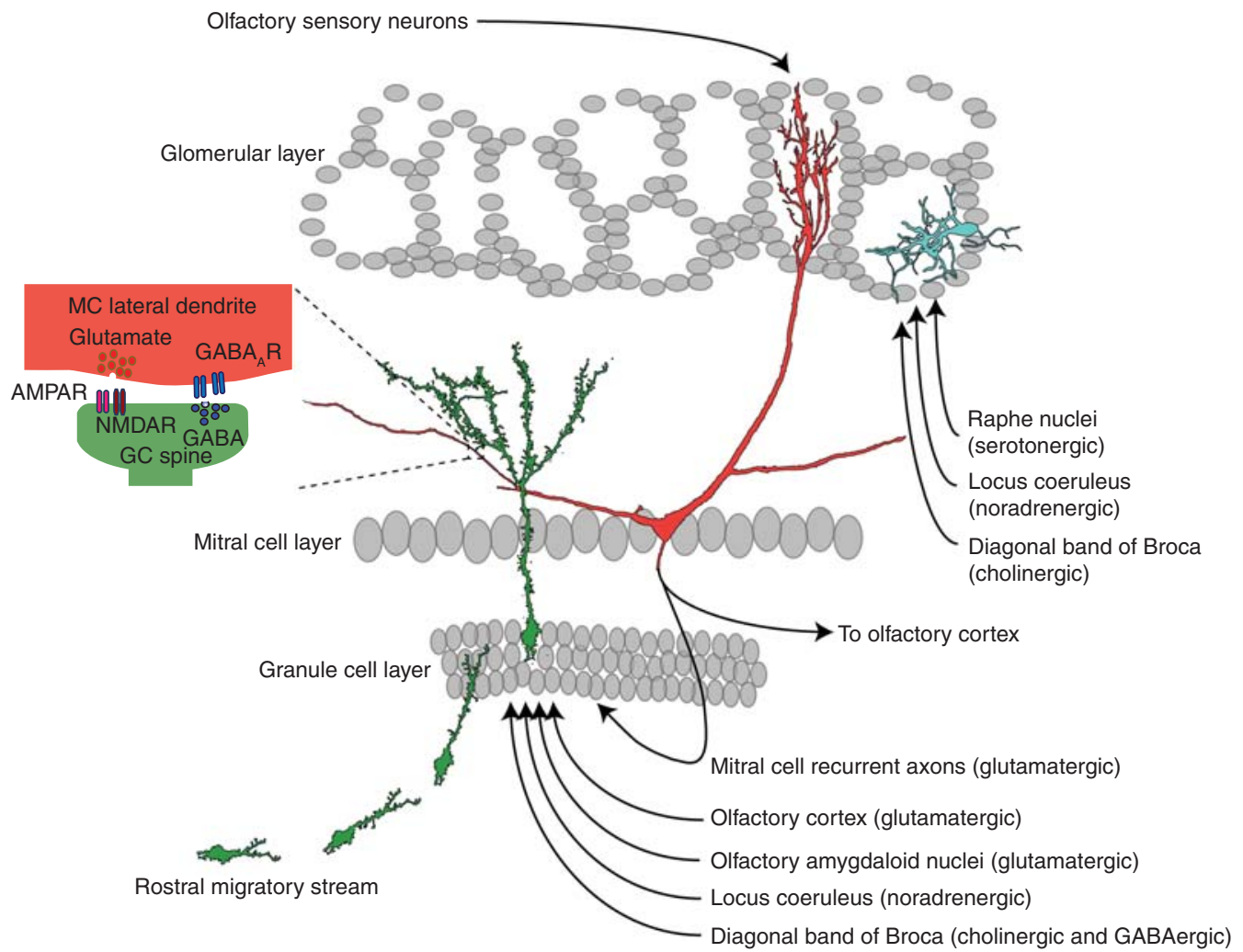

Figure 1. Major cell types in the rodent main olfactory bulb (OB). Periglomerular neurons (blue) and mitral/ tufted neurons (red) receive inputs from olfactory sensory neurons. Granule cell interneurons (green) form a unique reciprocal dendrodendritic synapse (see inset) with mitral/tufted neurons in which mitral/tufted neurons excite granule cells that can evoke reciprocal $\gamma$-aminobutyric acid (GABA)ergic inhibition onto the same neuron, or onto neighboring mitral/tufted neurons. Adult neurogenesis regenerates both periglomerular and granule cell interneurons throughout life. Both neuron types arrive into the $\mathrm{OB}$ as migrating progenitors in the rostral migratory stream, and they differentiate into their mature form within their target lamina. Centrifugal axons from numerous higher brain regions make synaptic contacts with interneurons, and the activity through these fibers is essential for the maturation and survival of adult-born neurons. 
P.-M. Lledo and M. Valley

cluding gain control, contrast enhancement, spatial decorrelation, and temporal synchronization (Kay and Stopfer 2006; Cleland 2010; Lepousez et al. 2013). In addition, many of these computations may be behaviorally modulated because of the large amount of top-down axonal fibers that carry contextual information to the $\mathrm{OB}$ (Fig. 1). Interneurons in the $\mathrm{OB}$ are central to this input-output transformation, and they represent a functionally diverse population that resides in every layer of the tissue.

The interneuron populations of the $\mathrm{OB}$ can be divided into two groups: those that surround the glomeruli (periglomerular [PG] interneurons), and the interneurons that reside in the mitral and granule cell layers (granule cell [GC] interneurons) (Shepherd 2003). PG interneurons are a diverse group and include both glutamateric and $\gamma$-aminobutyric acid (GABA)ergic neurons that mediate interactions within a glomuerlus, and between separate glomeruli. Their function is incompletely understood, but they likely help control the gain of glomerular inputs, and participate in lateral interactions between coactive glomeruli. They synapse with both mitral and tufted neurons and with each other producing multisynaptic loops within glomerular circuitry. In addition, PG neurons receive cholinergic, noradrenergic, and serotoninergic axonal inputs from higher brain regions that transmit information related to behavioral context (Fig. 1). Among the PG population, adult neurogenesis replenishes subtypes that express tyrosine hydroxylase, calretenin, and a fraction of calbindin glutamatergic interneurons.

GCs are by far the more populous of the OB interneurons, and they also appear to be more homogenous, at least according to classical immunohistochemical markers of interneuron diversity (DeFelipe et al. 2013). Granule cells play a key role in inducing and maintaining synchronization of projection neurons (Lledo and Lagier 2006; Lepousez and Lledo 2013), and projection neurons are the major target of GC inhibition. Granule cells are excited in two major ways. First, they receive excitatory input from the dendrites of mitral and tufted neurons. At this unique dendrodendritic synapse, both sides of the synaptic cleft have dual roles as pre- and postsynaptic structures. As a result, granule cells excited by a mitral or tufted cell may reciprocally release GABA across the same synapse, or from a neighboring synapse. Second, extensive systems of centrifugal fibers innervating the $\mathrm{OB}$ primarily contact local interneurons (Luskin and Price 1983). These centrifugal systems (Halász and Shepherd 1983) comprise excitatory inputs from the piriform cortex, the telencephalic basal ganglion, the magnocellular basal forebrain nuclei and the brain stem locus coeruleus (Shipley and Ennis 1996), and inhibitory inputs from the nucleus of the horizontal limb of the diagonal band (Kunze et al. 1992). Together, these top-down projections provide contextual information that formats odor codes according to specific behavioral demands.

\section{NEWBORN NEURONS IN THE ADULT OB: USE THEM OR LOSE THEM?}

A major theme that applies to adult neurogenesis in both the $\mathrm{OB}$ and in the hippocampus is how sensory experience strongly influences the maturation and survival of new neurons. In the OB, this was first suggested in an experiment in which sensory deprivation was found to reduce the number of granule cells through increased apoptosis (Corotto et al. 1994). Subsequently, it was shown that reopening the nostril following early occlusion (Cummings and Brunjes 1997) and olfactory enrichment in adults (Rochefort et al. 2002) could promote the survival of newly formed bulbar interneurons. These early studies gave rise to the insight that the rate of bulbar neurogenesis is determined not only by the constitutive rate of proliferation in the neurogenic niche, but also by cell death that occurs in the OB.

It is also possible that primary olfactory inputs indirectly regulate the early steps of neurogenesis, as apoptosis also occurs in the subventricular zone (SVZ) (Morshead and van der Kooy 1992). It has been reported that proliferation rates in the SVZ changed during olfactory deprivation in adults (Corotto et al. 1994), by surgical disruption of the rostral migratory stream (RMS) (Jankovski et al. 1998), and 
ablation of the OB (Kirschenbaum et al. 1999). Mandairon and colleagues (2003) have shown that cell death and proliferation increased in the SVZ, RMS, and OB during the first 2 weeks following olfactory deafferentation by axotomy and returned to control values 1 month after deafferentation. These changes closely followed the temporal pattern of olfactory sensory neuron death and regeneration, and they strongly suggest that cell apoptosis occurring throughout the SVZ-OB system influences all stages of adult neurogenesis. Thus, programmed cell death appears to be a widespread mechanism that regulates experience-induced changes in adult neurogenesis.

Sensory experience-dependent neuronal plasticity, occurring during a specific time window, is a crucial mechanism for establishing a finely tuned neuronal circuit in the developing brain. For example, deprivation of visual input from one eye shifts the response property of binocular zone neurons in the visual cortex preferentially toward the nondeprived eye input. This ocular dominance plasticity occurs during a sensitive period after the birth of animals, called the "critical period," during which monocular deprivation shifts ocular dominance (Hensch 2005). In OB adult neurogenesis, this rule applies as well. Yamaguchi and Mori (2005) found that the survival of newly generated neurons in the adult is sensitive to olfactory activity only during a restricted time window. They blocked olfactory activity by closing single mouse nostrils and found that survival was decreased the most by sensory deprivation 14 to 28 days after cell birth. This corresponds to the period of time when new neurons start receiving synaptic contacts, suggesting that new granule cells are susceptible to cell death in a sensoryexperience-dependent manner during the period of synapse formation. Interestingly, the same team subsequently showed that the elimination of adult-born GCs was promoted during a short time window in the postprandial period. They found that the number of apoptotic GCs specifically increased within a few hours after the start of feeding when following a restricted feeding period (Yokoyama et al. 2011). The origin of this apoptotic signal and the mechanism by which it is detected by young GCs are currently questions of great interest.

Perhaps one of the major functions of adult neurogenesis, acting in concert with centrifugal fibers, is to add plasticity of neuronal networks. This hypothesis is supported by experiments showing that sensory deprivation decreases the number, the dendritic length, and the spine density of newly generated interneurons (Saghatelyan et al. 2005; Kelsch et al. 2009). In addition, the top-down synaptic inputs received by immature GCs are likely reformatted by experience, at least in the proximal dendrite of the GC where sensory deprivation increases the number of synaptic inputs (Kelsch et al. 2009). Interestingly, these top-down connections also show increased long-term potentiation onto young granule cells 3 to 4 weeks after birth (Nissant et al. 2009), indicating that the input connectivity of new GCs is subject to both structural and functional synaptic plasticity. Long-term imaging of adult-generated GC dendrites has given powerful insights into activity-dependent structural dynamics (Livneh et al. 2009), showing that young $\mathrm{OB}$ interneurons are highly dynamic in contrast to projection neurons that show minimal structural plasticity (Mizrahi and Katz 2003). Despite increased structural plasticity in their critical window, even mature interneurons are morphologically dynamic (Livneh and Mizrahi 2012), indicating that the ability of adult-born interneurons to cause plasticity in the circuit is not restricted to their early stages of maturation.

It is noteworthy that bulbar neurogenesis occurs in a neuronal network in which sensory afferent inputs are also subjected to continual replacement. Mature olfactory sensory neurons have a limited life span ( $\sim 60$ days in rodents), which may be regulated by environmental factors (Watt et al. 2004). Bulbar neurogenesis might therefore be a mechanism for modulating sensory information processing in the brain in response to changes in the composition of the sensory epithelium. More experiments are needed to elucidate whether sensory neurogenesis and bulbar neurogenesis are tightly concerted, and whether sensory neuron generation influences changes in bulbar microcircuits. 
P.-M. Lledo and M. Valley

\section{DOES ADULT NEUROGENESIS SUPPORT LEARNING AND MEMORY?}

The role of sensory activity in the survival and death of adult-born neurons does not appear to be purely passive. Multiple studies now support a view that olfactory stimuli must evoke associations in higher brain areas for it to strongly modify adult-born neurons (Alonso et al. 2006; Mandairon et al. 2006; Mouret et al. 2008; Sultan et al. 2011). In particular, learning to discriminate between two odorants has been shown to reduce the elimination of newly generated neurons by apoptosis in their critical window (Petreanu and Alvarez-Buylla 2002; Winner et al. 2002; Giachino et al. 2005; Yamaguchi and Mori 2005). In one study, learning was found to not only control the survival of newly generated neurons, but also to regulate their spatial distribution (Alonso et al. 2006). Surprisingly, this effect was not linked to the activated region of the $\mathrm{OB}$ associated with the reward. Instead, survival rates were significantly higher in loci in which the activation driven by the nonreinforced cue was highest. It is thus possible that odor information without reward (i.e., negative information) plays a more dominant role in the survival of newly generated neurons than positive information in associative conditioning. Whatever the mechanism, a learning period of only 6 days is clearly sufficient for substantially better survival rates of newly generated neurons in some regions and not in others.

Why does the newly generated neuron population grow in some restricted areas, but not in others? It is possible that adjusting the distribution of newly generated neurons in a learning context enhances the contrast between spatial activation patterns produced by two odorants. This supports the hypothesis that small differences in regional pattern activity can be used for odorant discrimination as long as animals are subjected to the motivational and experimental consequences of differential reinforcement. The most recent results indicate that sensory inputs in a learning context are important for the local control of the survival of new neurons in behaviorally relevant networks in the adult OB. They also indicate that sensory experience shapes $\mathrm{OB}$ microcircuits beyond the simple modulation of existing synaptic connections.

Is adult neurogenesis necessary for normal olfactory learning? Several attempts have been made to test this question by disrupting adult neurogenesis using genetic disruption (Kim et al. 2007; Imayoshi et al. 2008; Sakamoto et al. 2011), X-ray irradiation (Lazarini et al. 2009; Valley et al. 2009), or chemical antimitotics (Breton-Provencher et al. 2009; Moreno et al. 2009; Sultan et al. 2010), to disrupt neuronalstem-cell proliferation. Unfortunately, these various approaches, each with its own limitations, have not converged on a single function for which adult neurogenesis is necessary.

In a recent study, adult-born neurons were specifically activated during a learning task, producing the first causal link between the activity of new neurons and odor discrimination learning and memory. Adult-born neurons were put under the control of the light-gated ion channel channelrhodopsin, and they were stimulated at different frequencies and at different timings relative to an odor-discrimination task (Alonso et al. 2012). This study found that the inhibition provided by a small fraction of mature adult-born neurons in the $\mathrm{OB}$ circuit was sufficient to accelerate discrimination learning and enhanced long-term memory. Interestingly, this stimulation did not alter the thresholds of detection for pure odorant molecules, nor did it alter the animal's fully trained capability for odor discrimination. In addition, this strong behavioral modulation was only provided by mature adult-born neurons and not by mature interneurons born just after birth of the animal. Thus, there was an immediate causal relationship between the activity of a small number of mature adult-born neurons and $\mathrm{OB}$ circuit function. This result raises many questions about the uniqueness of adult-born neurons, and how their influence on behavior can be so strong. In a follow-up study, a clue was found that could explain this unique behavioral effect (Valley et al. 2013). This study used channelrhodopsin to directly compare the inhibitory synaptic output of mature adult-born GCs to mature cells that were born when the animal 
was just a week old, and found that adult-born synapses were uniquely resistant to a form of synaptic depression mediated by the metabotropic $\mathrm{GABA}_{\mathrm{B}}$ receptor. This resistance increases the synaptic reliability of adult-born neurons, and may be one of several unique features that enable them to have a particularly strong influence on circuit function.

\section{ADULT NEUROGENESIS IN THE REAL WORLD: THE ETHOLOGICAL RELEVANCE OF CIRCUIT REMODELING}

Adult neurogenesis is a biological process that is conserved throughout evolution and thus likely has an adaptive function. But what advantages does bulbar neurogenesis give to an organism in the wild? Is it involved in survival, reproduction, or fitness? The physiological processes underlying adult neurogenesis are well studied; nevertheless, other than investigations reported in seminal papers in birds and chickadees, very few experiments have addressed its possible ethological functions.

Because of seasonal variation in the availability of food, some birds must rely on their spatial memory for food retrieval. The survival of these food-storing birds during the winter critically depends on their cache-recovery ability, and lesions of the hippocampus have been found to impair their spatial memory (Krebs et al. 1989). In addition, these species have larger hippocampus volumes than nonstoring birds (Healy and Krebs 1993). Large numbers of newly generated neurons are recruited to the hippocampal complex of free-ranging, adult, blackcapped chickadees during the fall, when the birds are storing food. This behavioral context strongly suggests that the recruitment of newly generated neurons is important for survival.

Recent studies have begun to explore olfactory adult neurogenesis in ethological contexts. For instance, Shingo et al. (2003) investigated bulbar neurogenesis during pregnancy and lactation in the mouse. They reported that the rate of neurogenesis increased in the first trimester of gestation, during the first week of lactation, and following mating. Of the various stimulating maternal hormones, prolactin (PRL) ap- peared to be a key factor triggering bulbar neurogenesis. During this critical first few weeks following birth, granule cells in the $\mathrm{OB}$ of lactating mothers also show increased structural stability, suggesting that the stable integration of increased numbers of interneurons is necessary for some aspect of maternal behavior (Kopel et al. 2012). OB adult neurogenesis also appears to play a role in mating behavior. The pheromones released by dominant male mice has been found to increase olfactory and hippocampal neurogenesis in females, and this increase in cell proliferation is necessary for female preference for dominant mates (Mak et al. 2007). Likewise, in male mice, the ability to recognize the smell of offspring relies on PRLstimulated neuronal proliferation (Mak and Weiss 2010).

Interestingly, disrupting adult neurogenesis does not appear to alter maternal behavior (Feierstein et al. 2010; but see Sakamoto et al. 2011). This is consistent with what is known about mouse nesting behavior. House mice nest communally in the wild and in the laboratory under seminatural conditions (Manning et al. 1992). In communal nests, several females give birth and nurse offspring and nonoffspring indiscriminately. Female mice nest communally with other females possessing a similar major histocompatibility complex; more generally, communal nesting partners tend to be kin (Manning et al. 1992). Even if mothers recognized their own pups, it is unlikely that in mixed-litter nests lactating females would nurse their pups and block access to nonoffspring. In terms of adaptive fitness, it is possible that mother mice reduce the cost of sharing milk with nonoffspring by nesting with closely related conspecifics (Hayes 2000).

\section{WHAT IS THE IMPORTANCE OF ADULT OB NEUROGENESIS IN HUMANS?}

One of the oldest beliefs about human perception is that we have a poor sense of smell. At first glance, this general belief seems to be grounded on scientific evidence, as previous human genetic studies showed a decline in the number of functional olfactory receptor genes during 
P.-M. Lledo and M. Valley

primate evolution to humans (Gilad et al. 2004). According to this view, the use of an arboreal habitat and the adoption of an erect posture during human evolution have led to the gradual increase in the importance of vision and decrease in the importance of smell. However, humans possess several overlooked olfactory features that may benefit their sense of smell including the structure of the nasal cavity, retronasal smell, increased olfactory cortical space, and the ability to use language to parse olfactory perception. After all, the relationship between genotype and phenotype is complex, and thus simply counting olfactory receptor genes is a poor indicator of olfactory performance. Therefore, if human smell perception is better than we thought, it may have conserved some of its original traits. Previous findings that human OBs incorporate newly generated neurons (Liu and Martin 2003; Bédard and Parent 2004; Curtis et al. 2012) may support this assumption. Newly generated neurons in humans may modulate olfactory discrimination in a similar way as that observed in rodents. This may come as a surprise to many people, although not to those who make their living using their noses, including enologists, perfumers, and food scientists. Anyone who has taken part in a wine tasting, or observed professional testing of food flavors or perfumes, knows that the human sense of smell has extraordinary capacities for discrimination and learning.

However, recent work casts doubt on the magnitude of adult neurogenesis in humans. In one study, the investigators characterize a loss of neuronal migration through the rostral migratory stream after infants are 18 months old (Sanai et al. 2011). In another study, longterm radiolabeling of adult-generated neurons in humans suggested that the rate of addition to the $\mathrm{OB}$ is very low compared with the hippocampus, at least in the cohort of psychiatric patients used in these studies (Bergmann et al. 2012; Spalding et al. 2013). Together, these data suggest that the canonical process as defined in rodents, that adult-born neurons originate in the subventricular germinal zone and migrate rostrally using chain migration to reach the $\mathrm{OB}$ circuitry, may not apply well to humans. In- stead, adult-born neurons in the human $\mathrm{OB}$ are likely to be locally generated and few in number, suggesting that there are significant limits to the magnitude of adult neurogenesis in the human OB. This identifies a fundamental difference in the plasticity of the human brain compared with other mammals. Although model organisms will necessarily continue to play a significant role in understanding neurodegenerative diseases, future research will need to take a more nuanced view of the differences between human and model olfactory systems.

One interesting lesson recently learned from rodent systems is that just a few adult-born neurons can have a large impact on olfactory circuits and olfactory behavior. In the most striking example, optogenetic stimulation of just a few thousand mouse interneurons was sufficient to add enough information to an olfactory precept to accelerate the rate of learning an olfactory discrimination (Alonso et al. 2012). It is thus important to avoid the fallacy that the number of a certain cell population is proportional to its functional importance. This may obviously apply to the human olfactory system, and gives hope that adult regeneration within this structure underlies some of our capability to learn and associate meaning to our olfactory world.

Time has seen the fall of a century-old dogma and the introduction of the new field of adult human neurogenesis. The priority for investigators in this field has shifted from documentation of the existence of newly generated neurons to understanding their functions. Many basic mechanisms regulating adult neurogenesis are still unknown. New neurons are generated continually in many regions of the adult central nervous system in nonmammalian vertebrates (Zupanc 2001). Thus, comparisons of adult neurogenesis in a broad range of species should yield new insights into the evolution and function of this process. A central question in the field of neurogenesis in the human olfactory system has yet to be answered. What functions might this well-conserved biological process have in humans?

Active neurogenesis in adult humans shows that the mature central nervous system has the 
capacity to regenerate, raising hopes that the nervous system, damaged by injury or degenerative neuronal diseases, can be repaired. Understanding the basic mechanisms regulating adult neurogenesis under normal and pathological conditions will provide the foundation for cellreplacement therapy, with either endogenous adult neural stem cells or transplanted cells from various sources. Technical advances have propelled progress in the field of adult neurogenesis. Aided by novel technologies, including in vivo dynamic imaging and optogenetics, the best is yet to come.

\section{CONCLUDING REMARKS}

The sense of smell has played an essential role during mammalian evolution. Odor representation is dynamic and highly complex, and may require unique plasticity mechanisms. Neuronal production, migration, and the replacement of mature interneurons in the adult OB represent some of these adaptive mechanisms taking place at different time scales.

We have seen that relay neurons of the mammalian OB circuit readily synchronize their firing and generate fast oscillations in response to olfactory nerve inputs. Because of its basic architecture and its synaptic organization, the local inhibitory network provides the $\mathrm{OB}$ with a unique form of inhibition that is crucial to induce and distribute rhythms to downstream olfactory structures. Inhibitory interneurons are involved in both the temporal and spatial organization of $\mathrm{OB}$ outputs. They transform the spatial dimension of the sensory information reaching the $\mathrm{OB}$ into spatiotemporal patterns. Continuous neurogenesis is therefore a mechanism by which the spatiotemporal coding is finely tuned. Such a mechanism may act together with experience-induced changes in synaptic weights, adding to the known range of adaptive properties of the adult OB.

This review has tackled two of the main problems related to the functional significance of adult neurogenesis. Both are fundamental issues. First, we have seen the role of adult neurogenesis in the context of OB function. Assuming that the bulb processes odor information before relaying it to the olfactory cortex, it can be hypothesized that adult neurogenesis enables the OB to adjust the degree of sensory information processing appropriately. Second, the existence of a pool of juvenile neurons, enabling the system to adapt to similar future situations, raises the possibility that adult neurogenesis acts "post hoc" to provide a structural basis for neuronal plasticity and learning. Research on adult neurogenesis in the olfactory system is thus not only intrinsically interesting, but also provides new avenues of exploration to increase our understanding of adult brain adaptation.

As a result of unprecedented developments in methods for examining the structure, development, function, and neurochemistry of olfactory circuits, research in olfactory plasticity has progressed substantially in recent years. Nevertheless, continued effort to develop new technologies, including those of molecular biology, neurophysiology, and functional imaging, will be necessary to unravel the mysteries of how neurogenesis shapes olfactory coding. After digging deeply into the fundamental basis of how the olfactory system processes sensory information, we are now starting to release exquisite fragrances of awareness.

\section{SUMMARY}

With a growing number of studies confirming that neural stem cells reside in the adult central nervous system, the function of newly generated neuronal cells in the adult brain is the source of intense research and debate. Adult neurogenesis is modulated by a wide variety of physiopathological conditions and environmental stimuli, offering the possibility that newly generated neuronal cells might be functionally associated with the response to these processes. Pioneering studies have proposed that newly generated neuronal cells support various brain functions, including learning, memory, and mood. However, more recent studies have begun to challenge these ideas. It is possible that adult neurogenesis alters the $\mathrm{OB}$ and hippocampus at the cellular, network, and system levels. Computational models and experimental approaches indicate that cell turnover might be especially 
beneficial for learning new information. In the $\mathrm{OB}$, it is clear that the number of new neurons increases after a learning task because newly generated neurons have longer life spans. Quite surprisingly, simple exposure to olfactory cues does not alter neurogenesis. Only in a learning context does sensory activation control the total number of newly generated neurons and refine their precise locations. These findings suggest that associative learning based on sensory stimuli presented with reward (or punishment) can modulate adult neurogenesis. In summary, adult-born interneurons act like transducers that integrate brain state information to tune sensory information processing in the OB.

\section{REFERENCES}

Abrous DN, Koehl M, Le Moal M. 2005. Adult neurogenesis: From precursors to network and physiology. Physiol Rev 85: 523-569.

Aimone JB, Deng W, Gage FH. 2011. Resolving new memories: A critical look at the dentate gyrus, adult neurogenesis, and pattern separation. Neuron 70: 589-596.

Alonso M, Viollet C, Gabellec MM, Meas-Yedid V, OlivoMarin JC, Lledo PM. 2006. Olfactory discrimination learning increases the survival of adult-born neurons in the olfactory bulb. J Neurosci 26: 10508-10513.

Alonso M, Lepousez G, Wagner S, Bardy C, Gabellec MM, Torquet N, Lledo PM. 2012. Activation of adult-born neurons facilitates learning and memory. Nat Neurosci 15: 897-904.

Bédard A, Parent A. 2004. Evidence of newly generated neurons in the human olfactory bulb. Brain Res Dev Brain Res 151: 159-168.

Bergmann O, Liebl J, Bernard S, Alkass K, Yeung MSY, Steier P, Kutschera W, Johnson L, Landén M, Druid H, et al. 2012. The age of olfactory bulb neurons in humans. Neuron 74: 634-639.

Breton-Provencher V, Lemasson M, Peralta MR, Saghatelyan A. 2009. Interneurons produced in adulthood are required for the normal functioning of the olfactory bulb network and for the execution of selected olfactory behaviors. J Neurosci 29: 15245-15257.

Cleland TA. 2010. Early transformations in odor representation. Trends Neurosci 33: 130-139.

Corotto FS, Henegar JR, Maruniak JA. 1994. Odor deprivation leads to reduced neurogenesis and reduced neuronal survival in the olfactory bulb of the adult mouse. Neuroscience 61: 739-744.

Cummings DM, Brunjes PC. 1997. The effects of variable periods of functional deprivation on olfactory bulb development in rats. Exp Neurol 148: 360-366.

Curtis MA, Low VF, Faull RLM. 2012. Neurogenesis and progenitor cells in the adult human brain: A comparison between hippocampal and subventricular progenitor proliferation. Dev Neurobiol 72: 990-1005.

DeFelipe J, López-Cruz PL, Benavides-Piccione R, Bielza C, Larrañaga P, Anderson S, Burkhalter A, Cauli B, Fairén A, Feldmeyer D, et al. 2013. New insights into the classification and nomenclature of cortical GABAergic interneurons. Nat Rev Neurosci 14: 202-216.

Feierstein CE, Lazarini F, Wagner S, Gabellec MM, de Chaumont F, Olivo-Marin JC, Boussin FD, Lledo PM, Gheusi G. 2010. Disruption of adult neurogenesis in the olfactory bulb affects social interaction but not maternal behavior. Front Behav Neurosci 4: 176.

Giachino C, De Marchis S, Giampietro C, Parlato R, Perroteau I, Schütz G, Fasolo A, Peretto P. 2005. cAMP response element-binding protein regulates differentiation and survival of newborn neurons in the olfactory bulb. $J$ Neurosci 25: 10105-10118.

Gilad Y, Wiebe V, Przeworski M, Lancet D, Pääbo S. 2004. Loss of olfactory receptor genes coincides with the acquisition of full trichromatic vision in primates. PLoS Biol 2: E5.

Halász N, Shepherd GM. 1983. Neurochemistry of the vertebrate olfactory bulb. Neuroscience 10: 579-619.

Hayes L. 2000. To nest communally or not to nest communally: A review of rodent communal nesting and nursing. Anim Behav 59: 677-688.

Healy SD, Krebs JR. 1993. Development of hippocampal specialisation in a food-storing bird. Behav Brain Res 53: $127-131$.

Hensch TK. 2005. Critical period plasticity in local cortical circuits. Nat Rev Neurosci 6: 877-888.

Imayoshi I, Sakamoto M, Ohtsuka T, Takao K, Miyakawa T, Yamaguchi M, Mori K, Ikeda T, Itohara S, Kageyama R. 2008. Roles of continuous neurogenesis in the structural and functional integrity of the adult forebrain. $\mathrm{Nat} \mathrm{Neu-}$ rosci 11: 1153-1161.

Jankovski A, Garcia C, Soriano E, Sotelo C. 1998. Proliferation, migration and differentiation of neuronal progenitor cells in the adult mouse subventricular zone surgically separated from its olfactory bulb. Eur J Neurosci 10: 3853-3868.

Kay LM, Stopfer M. 2006. Information processing in the olfactory systems of insects and vertebrates. Semin Cell Dev Biol 17: 433-442.

Kelsch W, Lin CW, Mosley CP, Lois C. 2009. A critical period for activity-dependent synaptic development during olfactory bulb adult neurogenesis. J Neurosci 29: 1185211858.

Kim WR, Kim Y, Eun B, Park O, Kim H, Kim K, Park CH, Vinsant S, Oppenheim RW, Sun W. 2007. Impaired migration in the rostral migratory stream but spared olfactory function after the elimination of programmed cell death in Bax knock-out mice. J Neurosci 27: 1439214403.

Kirschenbaum B, Doetsch F, Lois C, Alvarez-Buylla A. 1999. Adult subventricular zone neuronal precursors continue to proliferate and migrate in the absence of the olfactory bulb. J Neurosci 19: 2171-2180.

Kopel H, Schechtman E, Groysman M, Mizrahi A. 2012. Enhanced synaptic integration of adult-born neurons 
in the olfactory bulb of lactating mothers. J Neurosci 32: 7519-7527.

Krebs JR, Sherry DF, Healy SD, Perry VH, Vaccarino AL 1989. Hippocampal specialization of food-storing birds. Proc Natl Acad Sci 86: 1388-1392.

Kunze W, Shafton D, Kem RE, McKenzie JS. 1992. Intracellular responses of olfactory bulb granule cells to stimulating the horizontal diagonal band nucleus. Neuroscience 48: 363-369.

Lazarini F, Mouthon MA, Gheusi G, de Chaumont F, OlivoMarin JC, Lamarque S, Abrous DN, Boussin FD, Lledo PM. 2009. Cellular and behavioral effects of cranial irradiation of the subventricular zone in adult mice. PLoS ONE 4: e7017.

Lepousez G, Lledo PM. 2013. Odor discrimination requires proper olfactory fast oscillations in awake mice. Neuron 80: $1010-1024$.

Lepousez G, Valley MT, Lledo PM. 2013. The impact of adult neurogenesis on olfactory bulb circuits and computations. Annu Rev Physiol 75: 339-363.

Liu Z, Martin LJ. 2003. Olfactory bulb core is a rich source of neural progenitor and stem cells in adult rodent and human. J Comp Neurol 459: 368-391.

Livneh Y, Mizrahi A. 2012. Experience-dependent plasticity of mature adult-born neurons. Nat Neurosci 15: 26-28.

Livneh Y, Feinstein N, Klein M, Mizrahi A. 2009. Sensory input enhances synaptogenesis of adult-born neurons. $J$ Neurosci 29: 86-97.

Lledo PM, Lagier S. 2006. Adjusting neurophysiological computations in the adult olfactory bulb. Semin Cell Dev Biol 17: 443-453.

Luskin MB, Price JL. 1983. The topographic organization of associational fibers of the olfactory system in the rat, including centrifugal fibers to the olfactory bulb. $J$ Comp Neurol 216: 264-291.

Mak GK, Weiss S. 2010. Paternal recognition of adult offspring mediated by newly generated CNS neurons. Nat Neurosci 13: 753-758.

Mak GK, Enwere EK, Gregg C, Pakarainen T, Poutanen M, Huhtaniemi I, Weiss S. 2007. Male pheromone-stimulated neurogenesis in the adult female brain: Possible role in mating behavior. Nat Neurosci 10: 1003-1011.

Mandairon N. 2003. Deprivation of sensory inputs to the olfactory bulb up-regulates cell death and proliferation in the subventricular zone of adult mice. Neuroscience 119: 507-516.

Mandairon N, Stack C, Kiselycznyk C, Linster C. 2006. Enrichment to odors improves olfactory discrimination in adult rats. Behav Neurosci 120: 173-179.

Manning CJ, Wakeland EK, Potts WK. 1992. Communal nesting patterns in mice implicate MHC genes in kin recognition. Nature 360: 581-583.

Ming G, Song H. 2005. Adult neurogenesis in the mammalian central nervous system. Annu Rev Neurosci 28: 223250.

Ming GL, Song H. 2011. Adult neurogenesis in the mammalian brain: Significant answers and significant questions. Neuron 70: 687-702.

Mizrahi A, Katz LC. 2003. Dendritic stability in the adult olfactory bulb. Nat Neurosci 6: 1201-1207.
Moreno MM, Linster C, Escanilla O, Sacquet J, Didier A, Mandairon N. 2009. Olfactory perceptual learning requires adult neurogenesis. Proc Natl Acad Sci 106: 17980-17985.

Morshead CM, van der Kooy D. 1992. Postmitotic death is the fate of constitutively proliferating cells in the subependymal layer of the adult mouse brain. $J$ Neurosci 12: $249-256$.

Mouret A, Gheusi G, Gabellec MM, de Chaumont F, OlivoMarin JC, Lledo PM. 2008. Learning and survival of newly generated neurons: When time matters. J Neurosci 28: 11511-11516.

Nissant A, Bardy C, Katagiri H, Murray K, Lledo PM. 2009. Adult neurogenesis promotes synaptic plasticity in the olfactory bulb. Nat Neurosci 12: 728-730.

Petreanu L, Alvarez-Buylla A. 2002. Maturation and death of adult-born olfactory bulb granule neurons: Role of olfaction. J Neurosci 22: 6106-6113.

Rochefort C, Gheusi G, Vincent JD, Lledo PM. 2002. Enriched odor exposure increases the number of newborn neurons in the adult olfactory bulb and improves odor memory. J Neurosci 22: 2679-2689.

Saghatelyan A, Roux P, Migliore M, Rochefort C, Desmaisons D, Charneau P, Shepherd GM, Lledo P-M. 2005. Activity-dependent adjustments of the inhibitory network in the olfactory bulb following early postnatal deprivation. Neuron 46: 103-116.

Sakamoto M, Imayoshi I, Ohtsuka T, Yamaguchi M, Mori K, Kageyama R. 2011. Continuous neurogenesis in the adult forebrain is required for innate olfactory responses. Proc Natl Acad Sci 108: 8479-8484.

Sanai N, Nguyen T, Ihrie RA, Mirzadeh Z, Tsai HH, Wong M, Gupta N, Berger MS, Huang E, Garcia-Verdugo JM, et al. 2011. Corridors of migrating neurons in the human brain and their decline during infancy. Nature 478: 382 386.

Shepherd GM. 2003. The synaptic organization of the brain, 5th ed. Oxford University Press, New York.

Shingo T, Gregg C, Enwere E, Fujikawa H, Hassam R, Geary C, Cross JC, Weiss S. 2003. Pregnancy-stimulated neurogenesis in the adult female forebrain mediated by prolactin. Science 299: 117-120.

Shipley MT, Ennis M. 1996. Functional organization of olfactory system. J Neurobiol 30: 123-176.

Spalding KL, Bergmann O, Alkass K, Bernard S, Salehpour M, Huttner HB, Boström E, Westerlund I, Vial C, Buchholz BA, et al. 2013. Dynamics of hippocampal neurogenesis in adult humans. Cell 153: 1219-1227.

Sultan S, Mandairon N, Kermen F, Garcia S, Sacquet J, Didier A. 2010. Learning-dependent neurogenesis in the olfactory bulb determines long-term olfactory memory. FASEB J 24: 2355-2363.

Sultan S, Rey N, Sacquet J, Mandairon N, Didier A. 2011. Newborn neurons in the olfactory bulb selected for longterm survival through olfactory learning are prematurely suppressed when the olfactory memory is erased. J Neurosci 31: 14893-14898.

Valley MT, Mullen TR, Schultz LC, Sagdullaev BT, Firestein S. 2009. Ablation of mouse adult neurogenesis alters olfactory bulb structure and olfactory fear conditioning. Front Neurosci 3: 51. 
P.-M. Lledo and M. Valley

Valley MT, Henderson L, Inverso S, Lledo P-M. 2013. Adult neurogenesis produces neurons with unique GABAergic synapses in the olfactory bulb. J Neurosci 33: 1466014665.

Watt WC, Sakano H, Lee Z-Y, Reusch JE, Trinh K, Storm DR. 2004. Odorant stimulation enhances survival of olfactory sensory neurons via MAPK and CREB. Neuron 41: 95567.

Winner B, Cooper-Kuhn CM, Aigner R, Winkler J, Kuhn HG. 2002. Long-term survival and cell death of newly generated neurons in the adult rat olfactory bulb. Eur $J$ Neurosci 16: 1681-1689.
Yamaguchi M, Mori K. 2005. Critical period for sensory experience-dependent survival of newly generated granule cells in the adult mouse olfactory bulb. Proc Natl Acad Sci 102: 9697-9702.

Yokoyama TK, Mochimaru D, Murata K, Manabe H, Kobayakawa K, Kobayakawa R, Sakano H, Mori K, Yamaguchi M. 2011. Elimination of adult-born neurons in the olfactory bulb is promoted during the postprandial period. Neuron 71: 883-897.

Zupanc GK. 2001. A comparative approach towards the understanding of adult neurogenesis. Brain Behav Evol 58: $246-249$. 


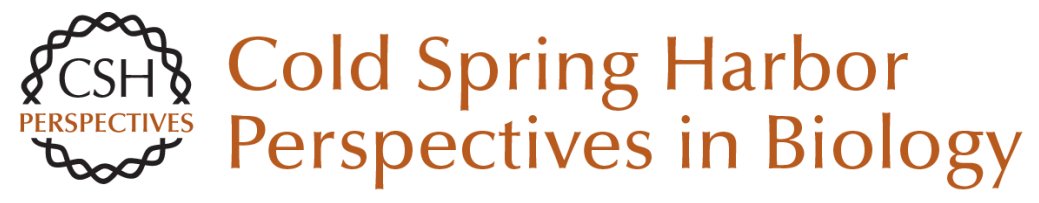

\section{Adult Olfactory Bulb Neurogenesis}

Pierre-Marie Lledo and Matt Valley

Cold Spring Harb Perspect Biol 2016; doi: 10.1101/cshperspect.a018945 originally published online May 27, 2016

\section{Subject Collection Neurogenesis}

Adult Neurogenesis and Psychiatric Disorders Eunchai Kang, Zhexing Wen, Hongjun Song, et al.

Neuronal Circuitry Mechanisms Regulating Adult Mammalian Neurogenesis Juan Song, Reid H.J. Olsen, Jiaqi Sun, et al.

Neurogenesis in the Developing and Adult Brain

--Similarities and Key Differences

Magdalena Götz, Masato Nakafuku and David Petrik

Genetics and Epigenetics in Adult Neurogenesis Jenny Hsieh and Xinyu Zhao

The Adult Ventricular-Subventricular Zone (V-SVZ) and Olfactory Bulb (OB) Neurogenesis Daniel A. Lim and Arturo Alvarez-Buylla

Diversity of Neural Precursors in the Adult Mammalian Brain Michael A. Bonaguidi, Ryan P. Stadel, Daniel A. Berg, et al.

Detection and Phenotypic Characterization of Adult Neurogenesis H. Georg Kuhn, Amelia J. Eisch, Kirsty Spalding, et al.

Maturation and Functional Integration of New Granule Cells into the Adult Hippocampus Nicolas Toni and Alejandro F. Schinder
Adult Olfactory Bulb Neurogenesis

Pierre-Marie Lledo and Matt Valley

Adult Neurogenesis in Fish Julia Ganz and Michael Brand

In Vitro Models for Neurogenesis Hassan Azari and Brent A. Reynolds

Engineering of Adult Neurogenesis and Gliogenesis

Benedikt Berninger and Sebastian Jessberger

Computational Modeling of Adult Neurogenesis James B. Aimone

Control of Adult Neurogenesis by Short-Range Morphogenic-Signaling Molecules Youngshik Choe, Samuel J. Pleasure and Helena Mira

Adult Neurogenesis: An Evolutionary Perspective Gerd Kempermann

Epilepsy and Adult Neurogenesis

Sebastian Jessberger and Jack M. Parent

For additional articles in this collection, see http://cshperspectives.cshlp.org/cgi/collection/

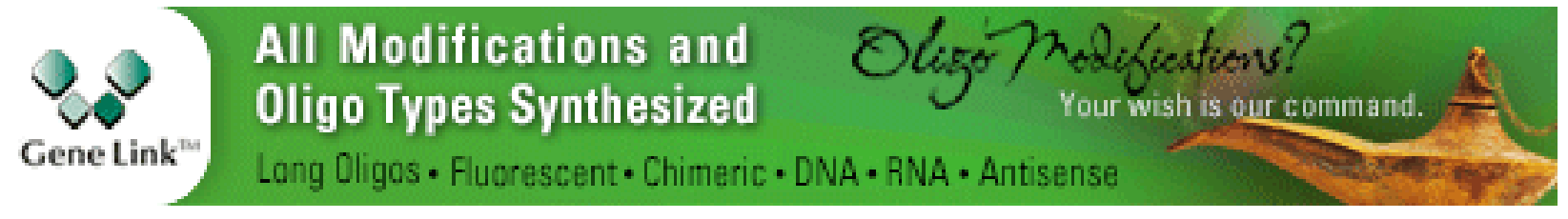


For additional articles in this collection, see http://cshperspectives.cshlp.org/cgi/collection/

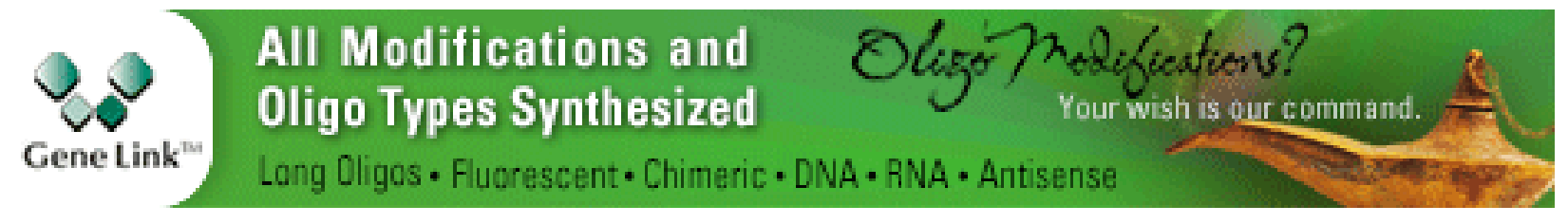

Copyright @ 2016 Cold Spring Harbor Laboratory Press; all rights reserved 